\title{
Programming in natural language: A descriptive analysis
}

\author{
MARC M. SEBRECHTS and PAUL H. GROSS \\ Wesleyan University, Middletown, Connecticut
}

\begin{abstract}
This study investigated the utility of natural language in specifying procedures. Performance of programmers was compared with that of nonprogrammers on two types of problems: a "realworld" ordering task and a computerlike database search task. Programmers performed better than nonprogrammers in general, although overall differences between the groups were greater for the real-world problem domain. Analyses of protocols suggest that an unconstrained natural language programming environment is presently infeasible. Although constraints imposed in the problem specification do appear to improve performance, they alone are not sufficient to produce efficient natural language programming. It is argued that programming requires general problemsolving strategies and that at least some aspects of such strategies may be dependent upon the specific language in which they are implemented.
\end{abstract}

Increasingly large numbers of people in a variety of professions are required to do some type of computer programming. As a consequence, it has become important to improve the ease with which programming can be learned and practiced. Numerous instructional manuals and debugging tools have been introduced to try to improve performance. One suggestion for the improvement of programming is the specification of procedures in natural language. In some cases, namely, in a number of database retrieval systems, natural language interfaces are already being used in place of previously complicated programming tools.

There are, of course, a number of advantages to natural language. The syntactic and semantic aspects of the language are already highly overlearned. Thus, it may be possible to focus on the problem-solving procedures rather than on implementation details. A number of people, however, have argued that programming requires an exact formalism that is beyond the precision of normal languate use (Shneiderman, 1980; Wirth, 1983). There is some empirical work that supports such reservations. Miller (1981) asked a group of nonprogrammers to write English solutions to several database problems; he found that, in general, there were problems with the way in which solutions were written that could not be resolved by an existing computerized natural language system.

The present study uses similar techniques to examine additional population and task characteristics that are relevant to the feasibility of natural language programming. In order to differentiate the language constraints from the

\footnotetext{
This research was supported by a project grant from Wesleyan University, a research grant from Digital Equipment Corporation, and NSF Grant IST82-17572. The views expressed are those of the authors and are not necessarily endorsed by Digital Equipment Corporation. Send correspondence to $M$. Sebrechts, Department of Psychology, Wesleyan University, Middletown, CT 06457.
}

conceptual skills that are a part of programming, this study compared performance of programmers with that of nonprogrammers. If the general procedural concepts are language-independent, then programmers should be able to use them in English as well as in the specific programming languages with which they are familiar.

In addition, it is important to know the extent to which solutions are constrained by problem definition. Subjects were therefore given two tasks: one that was computerbased (a modification of Miller's, 1981, database search task) and one that was not (organizing records to be recorded on cassettes). The different problem types made it possible to separate difficulties in using natural language in order to specify procedures from problems in understanding a particular domain or in mapping a procedural language to that domain.

Finally, the study was designed to provide a programlike set of task demands. Whereas Miller (1981) had required his subjects to write procedures for other people, our study stipulated that the person for whom the instructions were to be written had very limited skills. Any complex procedure had to be explained in detail. This was designed to eliminate the possibility that subjects were writing incomplete procedures because they expected the reader to fill in the missing pieces.

\section{METHOD}

\section{Subjects}

There were 20 subjects from the Wesleyan University community, including students, faculty, and staff. Half of the subjects had prior programming experience and were classified as programmers. All subjects in this group had taken at least three programming courses; they had had an average of 5.3 years of programming experience (range: 2-18 years) with an average of 4.3 programming languages (range: 2-8 languages). This group was repre- 
sentative of programmers with moderate experience; only two of the subjects would have been considered highly experienced programmers.

The other 10 subjects had had no programming experience, although many of them had had some experience with text editing. These subjects, called "nonprogrammers," were matched as closely as possible to the programmers for age, sex, and education.

\section{Procedure}

Subjects were tested individually. They were required to provide written instructions that someone else could read to solve two problems that had the following constraints: (1) The instructions were to be written in complete English sentences; (2) they were to be written at a level that could be understood by a person who had full comprehension of English, but who was capable of performing only "basic" tasks such as reading or finding a name in a list of names; (3) the procedure for problem solution had to be complete; and, (4) each instruction was to be written in a separate sentence.

Problems. There were two main problems presented to every subject. The first was from a "real-world" domain and required subjects to provide instructions for ordering records to be recorded onto cassettes. The second problem was typical of a computer database search task that required information to be extracted from a set of files. It employed the database structure used by Miller (1981), although the information to be extracted was different. Specifications for the two problems are outlined in Tables 1 and 2 . For both problems, subjects were given a detailed description of the task.

Table 1

Main characteristics of Problem 1

\section{Givens:}

Six record albums.

At least one record by each of four artists.

One album can be recorded on one side of a casette.

Task:

Write out the instructions for ordering the records such that when recorded in that order you will have as many cassettes with only one artist as possible.

Table 2

Main characteristics of Problem 2 (Adapted From Miller, 1981).

Givens:

Three company files with employee information:

File 1: Name; Hourly Wage; Hours Worked Last Pay Period: Deductions File 2: Name; Employee Number; Age; Marital Status

File 3: Employee Number; Job Title; Year Hired; Supervisor's Rating Files 1 and 2 are organized alphabetically by employee name.

File 3 is organized numerically by employee number.

\section{Task:}

Provide a detailed set of instructions for the following report: Make an alphabetized list of all employees rated superior. After each name you should include all information on this person except the rating. At the end of the report include the groups's average age, average salary, and average number of years with the company.
Table 3

The statement of Problem 3

These instructions describe to someone how to take the average of an unspecified number of integers in a list ending with 99999 . That last number is not to be averaged into the other numbers in the list. After reading the procedure carefully, fill in the missing instruction in line nine.

1) Start with a count of zero.

2) Start with a sum of zero.

3) Read in the first number.

4) If the number is not 99999, then do steps 6-9.

5) Go to step 10 .

6) Add the number to the sum.

7) Add 1 to the count.

8) Read the next number.

9)

10) Calculate the average by dividing the sum by the count.

11) Write down the average.

12) Done.

The subjects were provided with a pad of paper on which to write solutions to these problems as well as any "scratch work." The recording problem was always presented first, but there were no time limits imposed on writing. For each problem, the following times were recorded: reading instructions; reading problem directions; thinking time (recorded as the number of 5-sec pauses); and solution time.

Following the main problem-solution portion of the experiment, subjects were given a simple fill-in-the-blank test to evaluate their comprehension of a program written in English (Problem 3). They were presented with a 12-line English-language program that used a loop to take the average of a file of numbers, as shown in Table 3. The subjects were requested to fill in line number 9 . This approach is similar to one used by Soloway, Ehrlich, and Bonar (1982) to test knowledge of Pascal programs.

Subjects were then given a posttest questionnaire and were asked to evaluate the use of English and their problem-solving strategies.

\section{RESULTS AND DISCUSSION}

The first section of the results examines overall quantitative performance differences between programmers and nonprogrammers on the three problems. The second section focuses on qualitative analyses of the subjects' written responses.

\section{Overall Performance}

Natural language procedure comprehension. Problem 3 provides a rather simple measure of ability to comprehend a procedure written in English. Given the simplicity of the problem, it was reasonable to expect perfect performance, and the expectation proved to be correct for most of the subjects. Several of the nonprogrammers, however, wrote complex sentences, when a simple "Go to step 4" would have sufficed. In addition, three of the nonprogrammers had clearly erroneous solutions. Two subjects had a misconception of the problem; they gave 
the following answers: "Add number to the count" and " Add this next number to the sum and add 1." The third subject who made an error wrote, "If the number is not 99999 go to step 10"; this person understood that some type of transfer of control was necessary, but stipulated the wrong action for the specified test. Thus, even on a relatively straightforward problem, a natural language representation was not sufficient to ensure a correct solution.

Natural language procedure generation. Problems 1 and 2 provided more detailed descriptions of the types of "programs"' that people write in natural language. The adequacy of our subjects' solutions depended on whether or not the solutions were interpretable by the appropriate person or system. At present, there is no machine system that can successfully compile the programs written by our subjects. Since part of our goal, however, was to evaluate the relative difficulty of developing such a system, we tried to judge how close the solutions were to the type of natural language system defined in the problem statements. To this end, we developed a measure of overall performance that consisted of three components: (1) "completeness," which indicated whether or not all of the necessary steps were present and interpretable; (2) "level of assumption," which indicated whether or not the solution was unambiguous, given the description of the constraints on comprehension; (3) "adherence to directions," which indicated whether or not the subject had followed the general syntactic and semantic criteria that had been established. A 10-point scale was developed for each of these components. Each solution was then scored on these scales, and performance was measured as a composite of these three components.

Using this composite measure, programmers performed significantly better than nonprogrammers on the first problem $(t=2.63, p<.02)$. On the second problem, there was no significant difference $[\mathrm{t}(18)=1.31, \mathrm{p}>$ .20]. These effects seemed to be attributable to differences in both adherence to directions and level of assumption. On the first problem, from a "natural" context, programmers followed directions closely and made few inappropriate assumptions, whereas nonprogrammers showed less adherence to the problem constraints. In the second problem, the nonprogrammers generally showed substantial improvement in their adherence to the problem instructions, whereas the programmers increased their level of assumption about the domain.

The difference between problems was also reflected in the solution length. Programmers wrote substantially longer (mean of 287 words) solutions on Problem 1 than did nonprogrammers (134 words) $[t(18)=3.33, p<$ .01 ], whereas both groups showed equally long programs for Problem 2 [programmers: 377 words; nonprogrammers: 389 words; $t(18)=.14, p=.88$ ]

Apparently, subjects' assumptions are influenced by their familiarity with a domain as well as by their knowledge of procedures. Thus, nonprogrammers make fewer assumptions in the unfamiliar database domain than in the record domain; programmers use their knowledge about procedures to avoid excessive inference in the record problem.

Although none of the programs that the subjects wrote were entirely successful according to our criteria, several of the programmers' solutions came close to receiving perfect scores. Thus, with limited "debugging," a natural language understanding system with a restricted set of predefined procedures could "compile" some of these programs.

These global characteristics, however, do not capture the range of qualitative differences in the subjects' protocols. We next analyze the content of the written solutions in order to clarify some of the ways in which solutions that were successful and efficient differed from those that were not.

\section{Content Analysis}

In order to provide a general description of the types of constructs used by subjects, their responses were analyzed into six basic content categories suggested by Miller (1981): the use of existing data structures; the use of new data structures; attribute tests; transfer of control; use of general procedures; and comments. The number of occurrences of statements in each of these categories is presented in Table 4 . The first problem indicates rather different use of the various content categories by programmers and nonprogrammers. Programmers showed proportionately greater use of new data variables and transfer of control, whereas nonprogrammers made proportionately greater reference to general procedures and wrote more comments. On Problem 2, the distributions of content codes appeared to be more similar across the groups. This was consistent with the finding of greater overall performance similarity between programmers and nonprogrammers on the second problem.

Data structures and variables. As can be seen in Table 4, a substantial percentage of the subjects' statements for both Problems 1 and 2 made reference to data structures. On Problem 1, however, programmers used new data structures more often than did nonprogrammers. This reflected the tendency among nonprogrammers to assume that references could be disambiguated by domain knowledge.

There are also differences in how references were made. One way to refer is to declare a variable explicitly, using a name to which you can later refer. Thus, one subject wrote "Stack all albums in a pile, the input stack" and then later referred to "the input stack." Alternatively, a vague term or description can be used to refer. Another subject, for example, wrote "place it in a new pile" to describe the creation of a pile, and later referred to the same pile ("the new one") in a somewhat ambiguous way: "If there are [additional albums], place them in the new one."

Miller (1981) found no occurrence of explicit variable declaration. In the present study, there were numerous such declarations. In the first problem, only programmers 
Table 4

Numbers (and Percentages) of Different Types of Content Codes Used in Solving the Two Problems by Programmers and Nonprogrammers

\begin{tabular}{lccccc}
\hline & \multicolumn{2}{c}{ Problem 1 } & & \multicolumn{2}{c}{ Problem 2 } \\
\cline { 2 - 3 } \cline { 5 - 6 } Content Code & Programmers & Nonprogrammers & & Programmers & Nonprogrammers \\
\hline 1. Use of Existing Data Structures & $82(21)$ & $35(18)$ & & $102(20)$ & $125(26)$ \\
2. Use of New Data Structures & $121(31)$ & $37(19)$ & & $151(30)$ & $129(27)$ \\
3. Attribute Tests & $69(18)$ & $43(22)$ & & $79(15)$ & $55(11)$ \\
4. Transfer of Control & $74(19)$ & $24(12)$ & & $79(15)$ & $58(12)$ \\
5. General Procedure Use & $29(7)$ & $22(11)$ & & $85(17)$ & $87(18)$ \\
6. Comments & $19(5)$ & $33(17)$ & & $15(3)$ & $32(7)$ \\
\hline
\end{tabular}

used explicit variable declaration; in the second problem, both groups did. Explicit declaration was particularly evident in the context of finding an average, a context in which variables would be familiar from algebra. The following illustrates a nonprogrammer's use of variable declaration in Problem 2:

Count the number of employees on the list. Call this the Total Superior Employee Number (TSEN).

In sum, the programmers appeared to have a general variable declaration strategy, whereas the nonprogrammers seemed to have a procedure-dependent declaration strategy that was evoked by the computational demands of the second problem.

Both groups used labels to identify the results of an operation in the file problem. This was a useful strategy that decreased the need for complicated reference. It should, however, be noted, that specifying variables is not a simple matter; here, for example, the convention of placing parentheses around an acronym, "(TSEN)," is assumed to be sufficient for variable declaration.

Looping. Programmers used looping somewhat more frequently than nonprogrammers. For the first problem, this difference was significant $[\mathrm{t}(18)=2.38, \mathrm{p}=.03]$, reflecting the fact that programmers generally used one or two loops, whereas about half of the nonprogrammers did not use loops at all. On the second problem, both programmers and nonprogrammers used loops in their solutions, with no reliable difference in frequency of use.

The types of loops used by the two groups of subjects were quite different. Seven of the 10 programmers used a looping strategy that involved an initial setup of variables followed by a loop that incorporated the requisite changes for subsequent data. In programming, this is often required, since there are unique actions for the initial pass that need to be established outside a loop. This type of loop was not used by any of the nonprogrammers.

The types of loops used did not fit naturally with the options normally available in programming. Consider three types of loops in Pascal: FOR, REPEAT, and WHILE. The FOR loop specifies that the action should be executed a specified number of times. Our subjects tended to use "for" in combination with a terminating condition rather than with a specified number of repetitions. One subject, for example, wrote "for each album left, do the following." Pascal REPEAT loops test their condition at the bottom of the loop, and this same type of organization was frequent in our subjects' loops. Again, however, there was no well-defined structure to specify the form of the loop based on the syntax. More commonly, our subjects would start a loop with a "for" and then indicate the end of a loop with a "repeat" statement. A Pascal WHILE loop tests its termination condition first. Although Soloway, Ehrlich, Bonar, and Greenspan (1982) reported that novice programmers tend to use this kind of loop frequently, it was not used by any of our subjects. This may reflect the difficulty of that form of expression in natural language.

The following is an example of the way in which one of the programmers constructed a loop for Problem 2:

After completing the above, make a new list by doing the following. Take the first name on the old list ... cross this name off the old list. Go back to the top of the old list and start this process again, unless there are no names left in the old list. Ignore a name once it has been crossed off.

There a number of difficulties in trying to interpret these instructions. First, the phrase "start this process again" is difficult to disambiguate. The process had no specified beginning, and there are three other processes not listed here that take place between the first instruction ("Take the first name") and the last instruction ("Go back to the top of the old list"). In addition, there is another loop in the middle of this one that is also called a "process" in our subjects' protocol. Finally, the adequacy of the loop is closely tied to the ability to follow the transfer of control, another major source of difficulty in our subjects' procedures.

Transfer of control. One of the major concepts in the writing of any program is the specification of the sequencing of steps. Subjects frequently try to follow linear procedures, which are adequate for a number of simple tasks, such as the assembly of home appliances. However, in even the simplest programs, sequencing often deviates from a strictly linear order. Instead, the sequence of steps 
is determined by the conditions that obtain; and as programs become more general, more conditionals are required.

There were qualitative differences in the way transfer of control was expressed by programmers and nonprogrammers. All of the full "if-then-else" codes were written by programmers (usually in the following form: "if condition A occurs do B, otherwise do C"). Some of the nonprogrammers found alternative forms in which to express if-then-else conditionals. One subject, for example, produced the following:

3) If the word "superior" is shown, take that card out of the file.

4) Then go to the next card and repeat this procedure. Go to the next step.

5) If the word "superior" is not there, go on to the next card. Go to the next step.

There are a number of difficulties in comprehending these steps, including determination of the references and the termination conditions. However, this response does provide an explicit stipulation of both alternatives, that is, if the attribute is "superior" and if the attribute is not "superior." This type of conditional specification is similar to one used by Sime, Green, and Guest (1977). They compared a structure of the form IF-NOT-END (e.g., IF raining take_umbrella, NOT raining leave_umbrella, END raining) with the more common IF-THEN-ELSE structure. They found that subjects performed more accurately when using the IF-NOT-END form, at least for restricted short programs. Green (1977) argued that this result was due to the advantage of the explicit negation of "NOT" over the implicit negation of "ELSE." The spontaneous use of the IF-NOT form in our data supports the notion that the explicit negation is a more natural form for expression of conditionals.

In addition, all but one of the unconditional transferof-control codes were produced by the programmers (usually in a "Go to A" form). The one exception was a case in which a nonprogrammer explicitly instructed the reader to go to the next step at the end of each line.

It was evident for both groups that it was difficult to express transfer of control in English. Part of the reason for this is that there are no established conventions to indicate the beginnings and ends of blocks of procedures, as was evident in the protocol described in the above section on looping. In addition, there are established conventions in English that differ from those in standard programming languages. In the looping protocol quoted above, for example, the subjects writes "Go back to the top of the old list and start this process again," indicating the action to be performed. He or she then places qualifications on that action: "unless there are no names left in the old list. Ignore a name once it has been crossed off." Miller (1978) referred to this as action qualification. In contrast, programs usually state conditions prior to an action. Thus, our subject's response could be con- verted to something like the following: "If there are names left in the old list, then if any of those names are not crossed off, then go back to the top of the old list ...." This is what Miller called conditionalized action. These differences are important, since it is often difficult to clearly specify the end of an action qualification loop. If sentence boundaries are used to specify a loop in the above example, for instance, then a return would occur ("Go back to the top of the old list ....") without noting an important condition ("Ignore a name once it has been crossed off.') and the relationship between the programming context and subject's inferences.

Planning. Three measures provide an indication of the amount of planning subjects did: time spent prior to beginning writing, time spent thinking (delays between writing session), and scratch work. Both programmers and nonprogrammers waited only briefly before they began writing. On Problem 1, both groups spent an average of 3.75 min going over the directions. On Problem 2, the programmers spent $3.17 \mathrm{~min}$, and the nonprogrammers spent $2.40 \mathrm{~min}$.

Programmers spent approximately $25 \%$ of their solution time thinking about each problem. Nonprogrammers spent $29 \%$ of their solution time on Problem 1, and 35\% of their solution time on Problem 2 thinking. These times indicate that the solutions were not automatic, but required substantial cognitive effort. Nonetheless, planning appeared to occur in a linear sequence, since subjects did relatively little scratch work or advance organization of their solutions. Only six subjects did scratch work, and only two of these did their scratch work in any detail.

For these subjects, the programs represented an ongoing cognitive activity. They did not engage in careful advance planning in most cases, but thought about solution strategies as they solved the problem. To the extent that time allocation is a good indicator of cognitive strategy, these data suggest that problem solving occurs concurrently with specific implementations.

Strategy selection. Two types of strategies were available for Problem 2, a file-by-file strategy and a personby-person strategy (see Miller, 1981). The file-by-file strategy consists of extracting all the appropriate items from each file by searching every record in the file and then combining the results of these separate searches. The person-by-person strategy consists of searching for a person matching a particular attribute in one file; this is followed by checking whether there is a match on the appropriate attributes in the other files. This latter strategy, although it requires more complicated transfer of control, moving frequently between files, is more efficient in terms of the temporary creation of variables required by the fileby-file search. The person-by-person strategy was used by eight of the programmers, whereas it was used by only three of the nonprogrammers. One explanation for this discrepancy is that programmers have developed a "strategic plan" for efficient searches of databases whereas nonprogrammers use a file-by-file strategy that is more procedurally straightforward. 
The difficulties our subjects experienced with loops provide another indication of the types of strategies that are required to write effective programs. Many of our subjects appeared to have no clear concept of how to organize loop structures. This parallels some of the difficulties described by Soloway, Bonar, and Ehrlich (1983) in their studies of novice Pascal programmers. Pascal WHILE loops, for example, require a process-read strategy: The initial read is performed outside the loop; the loop then consists of processing a value and then reading the next value. This can be illustrated in the following format:

\section{Read first-value \\ While (test ith value) \\ Process ith value Read next-ith value \\ Process-read loop}

Novices find this concept difficult and often produce errors by following an inappropropriate read-process strategy in which the loop consists of a reading in of data followed by processing of the data internal to the loop. Since the data are read inside the loop, the terminating value is erroneously processed before the loop stops. Our results reflect a parallel difficulty with separating out some of the actions from the "natural" order of a loop. The programmers apparently did have some strategic plans than enabled them to formulate an implementation in English. The nonprogrammers, however, had not developed a strategic plan that fit the kind of implementation often used in programming, so they had no knowledge base from which to establish an English implementation.

Contextual references. One characteristic of natural language use is that many ambiguous terms or phrases are disambiguated by their context. Thus, we find comments such as "Copy all of the information associated with this name ..." or "If so, place them in the second pile ...." In each of these cases we have to identify the reference of terms such as "this name" in the first example or "them" in the second. Miller (1981) found that subjects had severe difficulty with referencing; $42 \%$ of the data references he identified required substantial semantic inference rather than the identification of a syntactic correspondence in the same or a preceding sentence.

In our data, there was substantially less difficulty with contextual referencing. Using Miller's categories, only $5 \%$ of the programmers' data references and $8.5 \%$ of those by the nonprogrammers were not disambiguated in the same or an adjacent sentence. Although the reason for this difference is uncertain, it may be due to the fact that our instructions required subjects to write for someone with only limited skills. The subjects' protocols indicate a conscious attention to detail. It should be noted, however, that avoiding potentially ambiguous sentences can result in very wordy and cumbersome constructions in English.

In addition, it is not only the ambiguity of individual sentences that is in question. A statement often functions as part of a more complex procedure. Thus, the subjects would introduce a comment such as "now you have four piles," which may appear unproblematic. In a normal programming context, this would in fact be a "superfluous" comment which would not affect the program. However, in the subjects' protocols, comments were often integral parts of the procedure; they provided necessary information to disambiguate other instructions.

\section{SUMMARY AND CONCLUSIONS}

The results reported here indicate that using natural language is not by itself sufficient to enable people to write good programs. Two of the subjects failed to correctly understand a simple 12-line program written in English (Problem 3). In addition, none of our subjects produced a program in English that was free of ambiguity within the constraints defined. This same ambiguity occurs in both normal correspondence (Miller, 1982) and in specification of database requests (Shneiderman, 1981). The difficulty of a natural language programming environment is not merely one of syntax; disambiguation often requires substantial domain knowledge, and if a truly general programming environment were to be created, the required knowledge would be extensive.

This does not, of course, rule out the possibility of natural language programming, but it does suggest that some strong constraints will be required in its implementation (see Rubinstein \& Hirsh, 1984, chap. 6). Requiring subjects to be precise can help to decrease ambiguity; thus, for example, our subjects made many fewer ambiguous pronominal references than did Miller's (1981), presumably because of the implicit constraint on reference imposed by our hypothetical unskilled reader. Problem-domain constraints can have a similar effect, as evidenced by the improved performance of the nonprogrammers on the database problem (Problem 2) compared with their performance on the recording problem (Problem 1).

As with current programming languages, the utility of natural language depends on the availability of strategic knowledge, as demonstrated by the comparison of programmers and nonprogrammerss. In an unfamiliar context (Problem 1), programmers produced fewer unwarranted assumptions. In general, there was positive transfer of previously learned skills. (See Reisner, 1977, for a related result on query languages.) Plans for the construction of loops and transfer of control, made the programmers' solutions generally more complete. In addition, for the second problem, the programmers generally chose a more efficient solution, whereas nonprogrammers chose a procedure that was less efficient, but somewhat simpler to describe.

Even programmers, however, had difficulties in adequately structuring their plans in English. This suggests that to some extent performance may be implementation specific, since we have confidence that the programmers would have solved the database problem easily in a programming language. 
Problem solving varies with the language used: An optimal BASIC solution may be a poor LISP solution. Likewise, if natural language programming were developed, specific implementation plans would have to be devised to take advantage of the characteristics of natural language. Whether or not positive transfer from the familiarity with natural language would overcome the potential negative transfer from the imprecision of normal usage remains an empirical question.

There are also some restrictions on the generalizability of the results presented here. First, the sample used covers only a limited range of potential programmers. Truly "naive" computer users or more experienced professional programmers may show somewhat different responses to natural language procedures (see Sheil, 1981). Nevertheless, a substantial number of programmers do programming work only occasionally (Shneiderman, 1980), and it is perhaps this group that is most likely to benefit from modifications to programming environments. The sample used here also scored well above average on measures of verbal and mathematical ability. It is important to investigate how different abilities influence the relative effectiveness of using natural versus formal languages to write procedures.

Second, the analyses reported here have described only a limited set of possible constraints on programming. One important potential constraint that was not examined is the form in which a program is presented; this aspect of programming can have major effects on performance (Fitter \& Green, 1981; Green, Sime, \& Fitter, 1981). Indentation for instance, can dramatically change the comprehensibility of nested conditionals. Natural language is undoubtedly subject to similar effects that will need to be taken into account in the design of any programming environment.

Third, the study examined the writing of only relatively simple programs. It does not address the question of program modifiability, and since the difficulty of programming is not a simple linear function of program length (Sheil, 1981), it may not reveal many of the problems associated with substantially longer programs.

It is important to address these questions for a more complete evaluation of natural language programming. However, even within the restricted domain examined, our results agree with those of Miller (1981) in suggesting that the use of natural language for programming has a number of severe limitations. The data extend his findings by suggesting that general strategic knowledge (as evidenced by programmers compared with nonprogram- mers) and performance constraints (as demonstrated by the effects of introducing an unskilled reader as an audience) can improve performance. An analysis of the character of that performance emphasizes the fact that programming is a "discipline" or a way of thinking (Dijkstra, 1976); any programming language, including a natural language, needs to be assessed in terms of its relative merits in aiding that cognitive activity.

\section{REFERENCES}

Dujstra, E. W. (1976). A discipline of programming. Englewood Cliffs, NJ: Prentice-Hall.

FitTer, M. J., \& Green, T. R. G. (1981). When do diagrams make good computer languages? In M. J. Coombs \& J. L. Alty (Eds.), Computing skills and the user interference. New York: Academic Press.

GreEN, T. R. G. (1977). Conditional program statements and their comprehensibility to professional programmers. Journal of Occupational Psychology, 50, 93-109.

Green, T. R. G., Sime, M. E., \& Fitter, M. J. (1981). The art of notation. In M. J. Coombs \& J. L. Alty (Eds.), Computing skills and the user interference. New York: Academic Press.

MILLER, L. A. (1978). Behavioral studies of the programming process. (IBM Research Report No. RC 7367). Yorktown Heights, NY: IBM Watson Research Center.

MrLler, L. A. (1981). Natural language programming: Styles, strategies, and contrasts. IBM Systems Journal, 20, 184-215.

Miller, L. A. (1982). Natural language texts are not necessarily grammatical and unambiguous. Or even complete (IBM Research Report No. RC 9441). Yorktown Heights, NY: IBM Watson Research Center.

REISNER, P. (1977). Use of psychological experimentation as an aid to development of a query language. IEEE Transactions on Software Engineering, SE-3(3), 218-229.

Rubinstein, R., \& Hersh, H. (1984). The human factor: Designing computer systems for people. Burlington, MA: Digital Press.

SHeIL, B. A. (1981). The psychological study of programming. Computing Surveys, 13, 101-120.

SHNEIDERMAN, B. (1980). Software psychology: Human factors in computer and information systems. Cambridge, MA: Winthrop.

ShNeIDERMAN, B. (1981). A note on human factors issues of natural language interaction with data-base systems. Information Systems, 6, 125-129.

Sime, M.E., Green, T. R. G., \& Guest, D. J. (1977). Scope marking in computer conditionals-a psychological evaluation. International Journal of Man-Machine Studies, 9, 107-118.

Soloway, E., Bonar, J., \& Ehrlich, K. (1983). Cognitive strategies and looping constructs: An empirical study. Communications of the $A C M, 26,853-860$.

Soloway, E., Bonar, J., \& Ehrlich, K. (1982). Cognitive strategies and looping programming knowledge. In Proceedings of the Conference on Human Factors in Computer Systems. New York: Association of Computing Machinery.

Soloway, E., Ehrlich, K., Bonar, J., \& Greenspan, J. (1982). What do novices know about programming? In A. Badre \& B. Shneiderman (Eds.), Directions in human/computer interaction. Norwood, NJ: Ablex.

WIRTH, N. (1983). Programming in Modula-2. New York: Springer. 